\title{
Considerations on Aircraft On-Ground De-Icing and Sustainable Airport Development
}

\author{
CORNEL DINU*, SORIN EUGEN ZAHARIA, CASANDRA VENERA PIETREANU \\ University Politehnica of Bucharest, Faculty of Aerospace Engineering, Department Aeronautical Engineering Systems and \\ Aviation Management, 1-7 Polizu Str., 011061, Bucharest, Romania
}

\begin{abstract}
On-ground de-icing activity represents a need to protect the aircraft under low-temperature operating conditions, with a high degree of saturation of suspended water vapor or in precipitation conditions that may favor depositions on aircraft surfaces. This activity determines the use of de-icing and anti-icing agents in significant quantities, which leads to compliance with environmental protection standards. In this paper the authors identify solutions for the management of the residues resulting from de-icing activities that lead to soil contamination with different chemical compounds from the fluids used for de-icing.
\end{abstract}

Keywords: deicing, contamination, chimical compounds, environmental protection

Protecting the environment as a result of human action on an industrial scale has become a necessity in the last 50 years due to the increasing rhythm of activities with a major impacton the environment. Although on-ground deicing activity has been for around 80 years, the impact of chemical compounds in the de-icing fluid on the ground and especially on the groundwater in the airport area, has become significant in recent years as a result of increased air traffic.

In this paper, the authors present their own methods for assessing the amount of aircraft de-icing fluid (ADF) and aircraft anti-icing fluid (AAF) at an airport, as well as the methods for reducing the impact of chemical compounds in these de-icing substances on soil and subsoil around the airport

The regulations in force such as the Law 107 / 25.09.1996 with further additions through H.G. 188 / 20.03.2002, H.G. 352 / 11.05.2005, but also by Community legislation: the Directive 91/692/EC - Discharge of Dangerous Substances in Water, the Directive 2000/60/EC - Water Framew ork, Council Directive 91/271/EEC - Urban Waste Water Treatment, 96/61/EC - Integral Pollution Prevention and Control, require rigorous control of the waste content in industrial activities.

Due to the specific content of substances used in the de-icing process, it is necessary to determine the load of biodegradable organic substances $\left(\mathrm{CBO}_{5}\right)$ and chemical oxygen demand by the potassium dichromate method (CCO-Cr), in accordance with SR ISO 6060/96. They must comply with the norms established when waste water is charged with pollutants, according to NTPA - 001 or 002 of H.G. 188/2002.

\section{Type of de-icing and anti-icing substances used}

The annual de-icing period may be between 3 and 5 months, depending on the geographic area in which this process takes place, as well as the weather conditions recorded during the cold period of the year. The de-icing process is of particular interest both in terms of positive effects on the safety of air operations (safety management), but also in terms of the direct impact on the environment.

The fluids used for on-ground de-icing the aircraft are diverse, depending on the severity of the environmental conditions, the degree of protection desired and the costs imposed by the technology used. According to the imposed requirements, the following types of de-icing/anti-icing agents are distinguished:

a) fluids type I ISO, containing a high amount of propylene glycol (aproximately $88 \%$ ), and the remaining is represented by corrosion inhibitors and dyes. These fluids have a degree of use of approx. $80 \%$ in the aeronautical industry [1]. In this category we list kilfrost, with its variants of use: kilfrost DF Plus - fluid based on triazol, kilfrost DF Sustain - fluid based on bio-derived glycol, kilfrost DF Plus (88) - a de-icing product developed exclusively for the North American market and kilfrost DF Plus (80) - fluid made exclusively for the Scandinavian market.

b) fluids type II ISO, which have a reduced amount of glycol of about $50 \%$ of the mass of the fluid. From this category we can mention: kilfrost $A B C-3$, kilfrost $A B C-K$ Plus, clariant Safewing MP II FLIGHT PLUS, ABAX Ecowing 26, clariant Safewing MP II FLIGHT and cryotech Polar Guard II.

c) fluids type III ISO are used for small aircrafts or commuter due to low adhesion to the applied surface, about 4 to 5 times lower than type II and IV fluids. For this reason, its use is very low compared to other types. From this category we mention: Safewing MP III 2031 ECO.

d) fluids type IV ISO are similar to Type II fluids, have a greenish color and require the use of more advanced pulverize systems, resulting in a longer period of protection of the surfaces. From this category can be mentioned: kilfrost $A B C-S$ Plus, ABAX Ecowing AD-49, Clariant Safewing MP IV LAUNCH, Clariant Safewing MP IV LAUNCH PLUS, Cryotech Polar Guard Advance, Dow UCAR ${ }^{\mathrm{T}}{ }^{\mathrm{M}}$ Endurance EG106 and Dow UCAR ${ }^{\mathrm{TM}}$ FlightGuard AD-49.

Considering this diversity of available aircraft de-icing fluid on the market and the constraints on their use as a safety measure against ice deposition on aircraft surfaces, a comparative analysis of the adverse effects on the environmentas a result of the compounds used is required. This analysis must take into account two aspects:

- the need to use it in this field, which is determined by the conditions imposed by aviation safety;

- long-term costs due to adverse effects on the environment, by soil and subsoil polluting in aeronautical service areas.

Regarding costs due to the need to carry out this process, they are accepted by all air carriers as a need for airline safety imposed by the regulations in the field, as part of the

\footnotetext{
* email: cornel_dinu@yahoo.co.uk; Phone: 0722552059
} 
safety policy established by all international regulatory bodies in aviation. Beyond these regulations, one should consider the consequences of not using these compounds and what can be expected. Throughout the history of deicing, numerous aviation accidents have been recorded, with material and human losses. In such situations, financial losses are immeasurable in relation to the value of the activities as a result of on-ground de-icing of aircrafts. So, while conducting this process and taking into account the smallest losses, one also needs to consider how to reduce the impact of these products on the environment. Therefore, the de-icing products must comply with the norms in force and must be carried out in accordance with the sustainable development policies of the environment, according to the law 310/2004, art. 2, lit. (I, m) and of the management plan of the area's hydrographic area.

For the correct assessment of the environmental impact, we need to consider the following:

- the amount of fluid used in the time unit;

- the place where this process takes place and how the resulting residue is collected;

- the type of fluid used and its chemical compounds.

\section{Experimental part}

\section{Methodology of research}

In this paper, the authors intend to identify the value of the traffic at Henri Coanda International Airport (LROP) and the amount of ADF / AAF consumed under simulated environmental conditions.

The research is based on the analytical assessment of the flight schedule offered by this airport and a series of data taken from a handling company, presenting the ADF/ AAF quantities resulting from different weather conditions during the winter season of 2017.

During the analyzed period, the traffic at this airport was reflected in 297 aircraft movements per day, of which 82.49 $\%$ were medium-sized aircraft such as the A320, B737 and Fokker 100 series, which denotes some homogeneity in traffic at this airport [2].
Another aspect identified in this research was that, out of the 175 statistical data recorded in January 2017, aeronautical agents used Type I and/or Type II ADFs in combination or separately [3].

\section{Results and discussions}

The evaluation of statistical data from this period presents a number of interesting aspects such as:

- the average temperature in January ranging from $-19^{0}$ $\mathrm{C}$ and $-1^{\circ} \mathrm{C}$, with an average value of $-8.25^{\circ} \mathrm{C}$ ( fig. 1 ).

- the temperature of the aircraft surface, a factor that depends on several variables such as: the temperature at flight level in cruise, time interval when the aircraft descends from the cruise level to ground level, the amount of fuel in the tanks, the material and structure of the wings, the stationary time on the platform untill de-icing. During the analyzed period, the aircraft surface temperatures varied from -35 to $9^{\circ} \mathrm{C}$, with an average value of $-15.76^{\circ} \mathrm{C}$, at an average wind intensity of $2.81 \mathrm{~m} / \mathrm{s}$. It is noted that during this period, the wind reached speeds of up to $11 \mathrm{~m} /$ $\mathrm{s}$, accentuating the phenomenon of decrease of the temperature on the measured surface. These values were determined at approx. 35 minutes after landing, and the aircraft had a metallic shell with the fuel tanks loaded at $70-80 \%$ of the capacity, (fig. 2).

- atmospheric humidity during the analyzed period had a variation between $75-100 \%$, with an average value of $93 \%$, in $48.65 \%$ of the studied cases. During this time, various weather conditions ranging from clear skies to low or even heavy snow, to overcooled rains were noted. All these aspects led to a layer of $1 \mathrm{~mm}$ on average, but there were situations where $100 \mathrm{~mm}$ values were recorded due to consistent snow of $500 \mathrm{~mm}$ when the aircraft stationed on the platform for several hours under conditions of consistent snow. Under these conditions, the ADF consumption is very high, reaching values of $2600-2800$ liters/aircraft. Such situations are not common during a season, but when they occur, large amounts of liquid are consumed within a very short time.

["C]

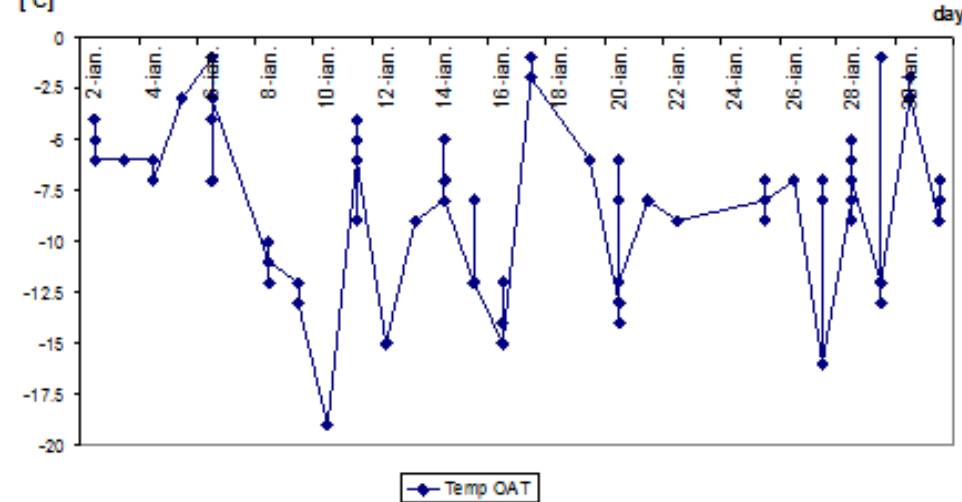

Fig. 1 Evolution of the environment temperature in January 2017

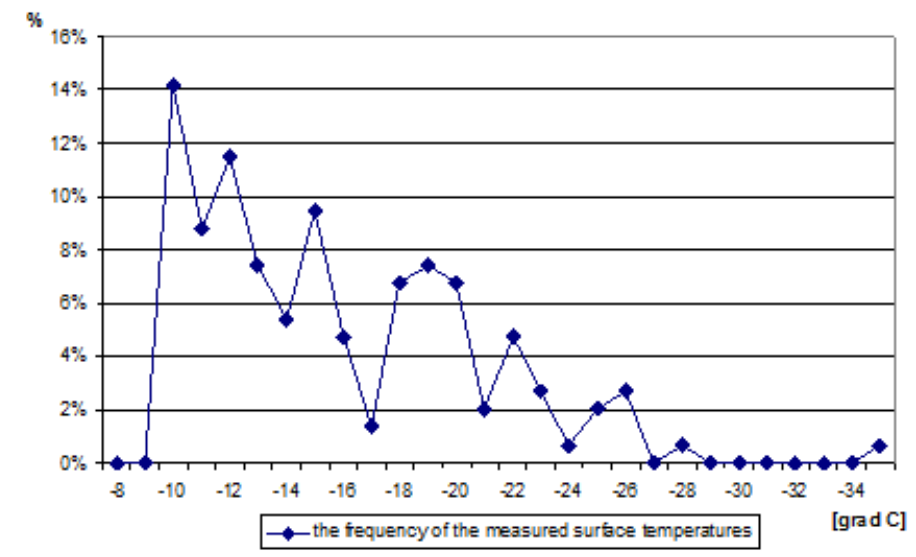

Fig. 2 Aircraft surface temperature measured before deicing 

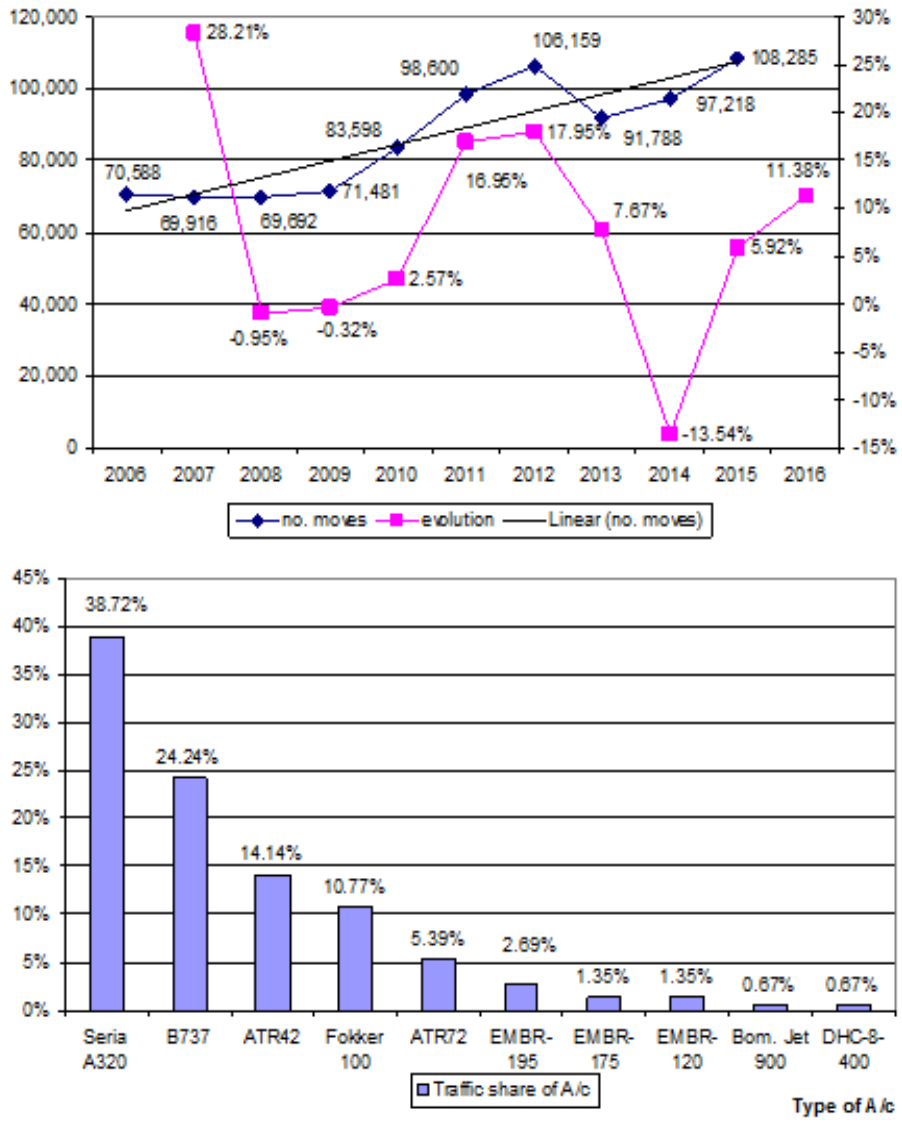

\begin{tabular}{|l|c|c|c|c|c|}
\hline $\begin{array}{c}\text { De-icing } \\
\text { surface }\end{array}$ & $\begin{array}{c}\text { Upper } \\
\text { wing }\end{array}$ & $\begin{array}{c}\text { Lower } \\
\text { wing }\end{array}$ & $\begin{array}{c}\text { Horizontal } \\
\text { empennage }\end{array}$ & $\begin{array}{c}\text { Vertical } \\
\text { empennage }\end{array}$ & Total surface \\
\hline Type A/c & {$\left[\mathrm{m}^{2}\right]$} & {$\left[\mathrm{m}^{2}\right]$} & {$\left[\mathrm{m}^{2}\right]$} & {$\left[\mathrm{m}^{2}\right]$} & {$\left[\mathrm{m}^{2}\right]$} \\
\hline A320 series & & 122.4 & 31 & 21.5 & $\mathbf{1 7 4 . 9 0}$ \\
\hline B737 & & 91.04 & 31.31 & 23.13 & $\mathbf{1 4 5 . 4 8}$ \\
\hline ATR 42-300 & 54.5 & & 12 & 8.3 & $\mathbf{7 4 . 8 0}$ \\
\hline Fokker 100 & 93.5 & & 21.72 & 12.3 & $\mathbf{1 2 7 . 5 2}$ \\
\hline ATR 72-500 & 61 & & 12 & 8.3 & $\mathbf{8 1 . 3 0}$ \\
\hline EMBR-195 & & 92.5 & 26 & 16.2 & 134.70 \\
\hline EMBR-175 & & 72.72 & 23.25 & 16.2 & $\mathbf{1 1 2 . 1 7}$ \\
\hline DHC-8-400 & & 271.9 & 51.1 & 23.6 & $\mathbf{3 4 6 . 6 0}$ \\
\hline
\end{tabular}

- the wind by its direction and intensity. During the analyzed period, a variation of the wind intensity from $0 \mathrm{~m} /$ $\mathrm{s}$ to $12.3 \mathrm{~m} / \mathrm{s}$ was noted, with an average value of $2.81 \mathrm{~m} /$ $s$ in almost $68.6 \%$ of the analyzed cases.

Considering these conditions, we have all the premises for an estimated calculation of ADF / AAF consumption at LROP airport, from the $1^{\text {st }}$ to $31^{\text {st }}$ of J anuary 2017 and the estimated ADF / AAF consumption throughout the winter of 2016-2017 at this airport.

In the case of LROP airport, the number of movements increased in the last 3 years with an average of $8.65 \%$, reaching 108,285 movements in 2016 and an average of over 6.9 thousand movements/month during the winter period (fig. 3). This shows a growing potential, even though there have been negative fluctuations in some years.

Analyzing the traffic structure at this airport, it is noticed that the DHC-8-400 aircraft has a double surface compared to the $\mathrm{A} 320$ series, the aircraft that forms the basis of this study (table 1). But if we look at the number of movements per day we notice that the DHC-8-400 operates just twice, which does not significantly affect the outcome of our
Fig. 3 Traffic evolution on LROP airport in 2006 2016 period

Fig. 4 Aircraft in operation share on LROP airport

Table 1

AIRCRAFT DE-ICING SURFACE analysis. The rest of the aircrafts, such as ATR 42/72, DHC$8-400$, EMBR 120,175 , account for $17.51 \%$ of the traffic at this airport, (fig. 4).

If we take into account the average values of the weather phenomena recorded during this period and make a simple calculation for each type of aircraft $(A / C)$, we can see that average quantities of 100,000 liters of ADF per day can be reached, (table 2).

Surely, this calculation is hypothetical, because over a day, the weather conditions may change, just as the concentrations chosen by each crew may be different. But the research aims want to show that there are important quantities distributed differently over time due to aircraft operating hours, which leads to a more detailed study of how much time is being used at peak hours and in severe weather conditions. In this respect, in severe weather conditions, which usually occur in the early hours of the morning, coinciding with the peak hour of operating at this airport, a number of 18-20 aircraft are identified, of which 12-14 are aircrafts of medium size. Under these circumstances, an average consumption/peak time of 
Table 2

QUANTITY OF AIRCRAFT FLUID, TYPE II, CONSUMED AT DE-ICING/ANTI-ICING

\begin{tabular}{|c|c|c|c|c|c|c|c|}
\hline Evaluation & $\begin{array}{l}\text { Quantity of } \\
\text { ADF type II }\end{array}$ & $\begin{array}{l}\text { Quantity of } \\
\text { ADF type II }\end{array}$ & $\begin{array}{l}\text { Quantity of } \\
\text { AAF type II }\end{array}$ & $\begin{array}{l}\text { Medium } \\
\mathrm{nr} \text {. of } \mathrm{A} / \mathrm{c}\end{array}$ & \multicolumn{3}{|c|}{ Total ADF/AAF consumption per A/c type } \\
\hline A/c type & {$[$ liters] $/ \mathrm{A} / \mathrm{c}$} & [liters]/A/c & [liters] / A/c & & [liters]/day & [liters] / day & [liters] / day \\
\hline A320 series & 455.2 & 358.9 & 534.0 & 115 & 52.351 & 41.271 & 61.413 \\
\hline B737 & 378.7 & 298.5 & 444.2 & 72 & 27,263 & 21.493 & 31.982 \\
\hline ATR42-300 & 194.7 & 153.5 & 228.4 & 42 & 8.177 & 6.446 & 9.592 \\
\hline Folkker 100 & 331.9 & 261.7 & 389.4 & 32 & 10.621 & 8.373 & 12.460 \\
\hline ATR72-500 & 211.6 & 166.8 & 248.2 & 16 & 3.386 & 2.669 & 3.972 \\
\hline EMBR-195 & 350.6 & 276.4 & 411.3 & 8 & 2.805 & 2.211 & 3.290 \\
\hline EMBR-175 & 292.0 & 230.2 & 342.5 & 4 & 1.168 & 921 & 1.370 \\
\hline DHC-8-400 & 902.1 & 711.2 & $1,058.3$ & 2 & 1.804 & 1.422 & 2.117 \\
\hline & & & & Total & 107.575 & 84.806 & 126.197 \\
\hline
\end{tabular}

15,000-18,000 liters may be reached. These results are useful for sizing ADF/AAF collector channels.

There is a large volume of ADF/AAF and an even more important aspect is that some handlers use aircraft deicing fluid (ADF) type I and aircraft anti-icing fluid (AAF) type II products, while others use only ADF/AAF type II.

The differences between these types of fluids are significant and are determined by the amount of base compound in the fluid mass, such as: EG-ethylene glycol, DG-diethylene glycol or PG-propylene glycol, for example [4]:

- Propylene glycol 20 - 85\%, Glycerol 0 - 40\%, PDO 0 $50 \%$, Xylitol 0 - 30\%, Non-NPE $0.025-0.40 \%$, Water 39 79.5\%; Policarboxid $0.03-0.4 \%$; Chelating agent 0.025 $0.2 \%$; Non-triazole corrosion inhibitor $0-0.08 \%$.

Regardless of the propylene glycol concentration, studies on its toxicity to the environment [5] have seen some tolerable limits tolerated by aquatic life in rivers near industrial sites. Of particular importance are benziltriazole and tolytriazole which are corrosion inhibitors, toxic and non-biodegradable, persisting in the environment [6]. According to the AMS standard 1428, if these substances exceed $50 \%$ of the established concentration, values higher than $1000 \mathrm{mg} / \mathrm{L}$, these substances may have a toxic effect on aquatic life. Among the beings considered as marks in the water quality assessment we list: daphnia and fathead minnows. If these contamination are evaluated on a permanent basis and reported not only to the regulations in force [7], butalso to a standard that allows for sustainable airport development, in close connection with an environmental strategy over a long period of time, it will lead to notable results towards environmental conservation.

\section{Means of environmental protection}

The development of the de-icing activity has led to the emergence of a variety of ADF/AAF fluids required by aircraft safety conditions by providing the best possible protection time. This has led to the use of different chemical compounds with different environmental impacts. Their use requires the establishment of safety measures in operation that respond to established environmental protection policies, such as:

- aircraft de-icing on a specially designed platform;

- integral collect of the fluids used for de-icing/anti-icing depending on fluid types;
- collecting the products resulting from the de-icing of the aircraft runways and taxiways.

Selective collection of these substances in special tanks is one of the solutions suitable for recycling the compounds used. Where this activity is based on the use of one type of ADF, the solution is simple because it allows the capture and storage of these compounds with ease. However, there are handling operators using two different types of ADF/ AAF, which complicates the technical solution for capture and storage.

By building a single de-icing platform between the taxiways $\mathrm{N}$ and $\mathrm{G}$, a selective and integral collection of the substances used in this process would be allowed, (fig. 5). This platform should allow simultaneous de-icing of 3 longhaul transport aircrafts (A340-200/MD-11/B 747-600), 4 medium-haul transport aircrafts (B757-200/A330/A300 type) or 8 short-haul transport aircrafts (type B737/A320/ Fokker 100). As a result of the collection of de-icing substances from this platform, but also from the entire surface, these residues can be decanted into special pools located in the vicinity of the airport, such as the case Buffalo Niagara International Airport (BNIA) [8].

The system is based on the existence of a network of collector pipelines in the de-icing platform infrastructure and an electrovalve system that allows controlled drainage of fluids, depending on the concentration and type of ADF/ AAF used, to the collecting tanks. This way, the collection and treatment of these wastes would allow for better recycling of de-icing/anti-icing fluids and would reduce the

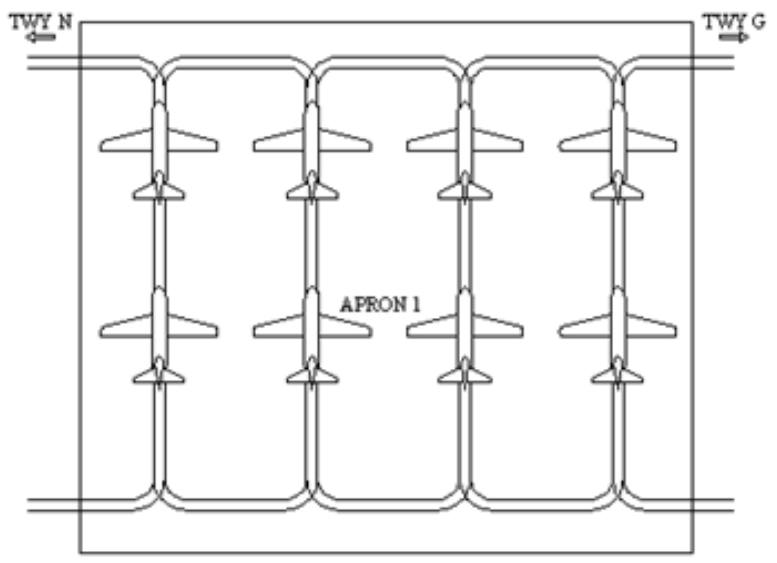

rIg. $\supset$ De-Icıng plattorm 
impact of chemical compounds found in the substances used.

Through global waste water indicators, it can be determined:Biochemical Oxygen Demand at 5 days (BOD5), chemical oxygen demand determined by the potassium dichromate method (CCO-Cr) or potassium permanganate (CCO-Mn), suspension materials (MS). With the help of these indicators we can identify the amount of pollutants present in the waste water discharged into the sewerage network, that must comply with the norms established by the legislation in force, see NTPA - 001 of H.G. 188/2002.

\section{Conclusions}

By reorganizing the de-icing activity as a result of building a specially designed platform on which this process will take place, a more efficient divider collection system can be achieved. The results of such an investment could have a good impact over time on the natural receiver, the Pasare River, but also on the urban waste water treatment plant.

If we take into account that the resulting sludge can be conditioned for use as a fertilizer in agro-culture, then it means that collateral benefits can also be achieved. But the most important aspect is stopping some chemical compounds, (such as triazole) to get into the underground water or into the sewerage network of the city. Through a periodic sampling from critical areas such as taxiways, hold positions and from around the platforms, the concentration of chemical compounds in the airport soil can be determined [9].

\section{References}

1. *** Estimates by The Dow Chemical Company;

2. *** The operating program of Henri Coanda International Airport for the winter of the years 2016 - 2017;

3. *** Company statistics of Romaine Airport Services;

4. SATYA P. CHAUHAN, MELISSA S. ROSHON, WILLIAM D. SAMUEIS, Compositions for deicing/anti-icing, PatentUS9080092 B2, no. demand US 14/025, 261, published on 14 July 2015;

5. *** Dow France S.A.S., Fiche de Donnees de Securite, Nom du produit: Propylene Glycol Industrial Grade, Version: 10.0, Date de révision: 12.06.2017;

6. ANNE SEELAND, MATTHIAS OETKEN, ALIZ KISS, ELKE FRIES, J ORG OEHLMANN, Acute and chronic toxicity of benzotriazoles to aquatic organisms, Environment Science Pollution Results, 2012, 19:1781 1790, DOI: 10.1007/s 1356-011-0705-z;

7. *** European Commission Regulations no. 1907/2006. Law 107/ 1996, Water Law, published in the Official Gazette no. 244 of 8 October 1996 and supplemented with: - EGG. no. 3/2010, published in the Official Gazette no. 114/2010 of 19.02.2010 and - EGG. no. 64/2011, published in the Official Gazette no. 461/2011, dated 29.06.2011.

8. *** Propylene Glycol Disposal, Buffalo Airport Deicing Fluid Treatment System, Buffalo Niagara International Airport, New York (United States); FAA, Buffalo Niagara International Airport, Airport master Plan update/CHEEKTOWAGA, Erie County, 30 aug. 2010, pag. 9;

9. CANCILLA A. DEVON, BAIRD J.C., ROSA R., Detection of Aircraft Deicing Additives in Groundwater and Soil Samples from Fairchild Air Force Base, a Small to Moderate User of Deicing Fluids, Bull. Environmental Contamination and Toxicology (2003) 70:868-875, Springer-Verlag New York Inc., DOI: 10.1007/s00128-003-0063-8.

Manuscript received: 6.07 .2018 